\title{
Measurements of Second-Order Properties of Point Processes
}

\author{
Bernard Picinbono, Life Fellow, IEEE
}

\begin{abstract}
The second-order statistical properties of point processes (PPs) are described by the coincidence function which can be measured by a coincidence device, but such measurements are long and complicated. We propose another method of measurement, and we analyze its performances. The starting point is that the coincidence function can be deduced from the probability density functions of the life times (the distances between points) of the process. The idea is to transform the PP into a positive signal whose values are these distances. From an appropriate processing of this signal, we deduce the coincidence function. For the validation of the method, we use PPs for which the coincidence function is known. The agreement between theory and experiment is, in general, excellent. Finally, the method is applied to measure the coincidence functions of some PPs for which no theoretical result is available.
\end{abstract}

Index Terms-Point processes (PPs), signal processing, signal representation, statistical measurements.

\section{INTRODUCTION}

$\mathbf{P}$ OINT PROCESSES (PPs) play an important role in many areas of physics, statistics, and engineering sciences. For example, in optical communications at low levels, the only available information is the set of random instants at which photons are detected. Similarly, the time instants at which telephone calls arrive at a switching center are a PP.

A PP is a random distribution of points in a space. If this space is the time axis, we have a time PP, and its random points $P_{i}$ 's are time instants $t_{i}$, which are sometimes called the events. All the PPs considered next are time PPs. In what follows, we consider only stationary PPs characterized by the fact that their statistical properties are invariant in time.

The complete statistical description of a PP is very complicated, and in most applications, one is obliged to use only first- or second-order statistical properties. The first-order description of a stationary PP is contained in its density $\lambda$, which is the average number of points per unit of time. The transposition to PPs of the concepts of the correlation function requires a specific analysis. The first attempt to describe the second-order properties of PPs was presented by Bartlett [1], [2]. Since a PP has no correlation function, the idea was to introduce a similar function, which was called the correlation density function.

Manuscript received January 26, 2007; revised May 31, 2007

The author is with the Laboratoire des Signaux et Systèmes (L2S), which is a joint laboratory of the Centre National de Ia Recherche Scientifique and the École Supérieure d'Électricité, 91192 Paris, France, associated with the University of Paris-Sud, Orsay, France (e-mail: bernard.picinbono@1ss. supelec.fr).

Color versions of one or more of the figures in this paper are available online at http://ieeexplore.iee.org.

Digital Object Identifier 10.1109/TIM.2007.911690
More recently, it was indicated that this function also appears in the description of a PP by coincidence analysis [3], and this is the reason to use the term coincidence function [4] which contains all the second-order properties of a PP.

The coincidence function can be measured by a coincidence device, a system widely used in nuclear physics. The measurements, however, are long and complicated and not adapted to various PPs. We propose another approach which does not start directly from the definition of the coincidence function but from its relation to the properties of the distances between successive points of the PP, which are sometimes called the life times [5]-[7]. By using the standard vocabulary of experimental nuclear physics, the device, in order to measure the coincidence function, is then no longer a coincidence circuit but a time-toamplitude converter (TAC) which transforms distances between points into pulses of amplitude proportional to these distances. These amplitudes constitute a discrete-time positive signal. By an appropriate statistical signal processing of these distances, it is possible to measure the coincidence function.

Coincidence function and coincidence measurements are at the basis of some applications in statistical optics and, more recently, in quantum communication [4], [8], [9]. For example, the structure of the coincidence function is a tool to decide whether an optical field is of classical or of nonclassical nature [8].

In Section II, we present a short review of properties of coincidence functions, and we describe the principle of their measurement from the analysis of life times. We theoretically discuss the precision of the method and its limitations. In Section III, we test the method by using computer experiments. There are some PPs for which the coincidence function can be theoretically calculated. By making measurements on those PPs, we can validate the behavior of the method and evaluate its precision. In general, the experimental measurements are in excellent agreement with theoretical results. In the last section, we apply the method in the case of some PPs for which the calculation of the coincidence function is almost impossible, and we present and discuss some examples.

\section{Definition and Measurement of the COINCIDENCE FUNCTION}

Let $N(t)$ be the number of points of a PP in the interval [0, $t$, where 0 is an arbitrary origin of time. The increment $d N(\theta)=$ $N(\theta+d \theta)-N(\theta)$ is the number of points in the interval $[\theta, \theta+d \theta[$. The second-order properties of a PP are characterized by its coincidence function $c(t)$, which is defined by

$$
\mathrm{E}\left[d N(\theta) d N\left(\theta^{\prime}\right)\right]=c\left(\theta-\theta^{\prime}\right) d \theta d \theta^{\prime} .
$$


The name comes from the fact that this function is generated by a coincident event. Indeed, a coincidence at $\theta$ and $\theta^{\prime}$ is the event defined by $[d N(\theta)=1] \cap\left[d N\left(\theta^{\prime}\right)=1\right]$. For regular PPs, which are the only ones studied in this paper, the increment $d N(\theta)$ takes only the values zero or one when $d \theta$ tends to zero. As a consequence, the expectation value appearing in (1) is the coincidence probability. The function $b(t)=c(t) / \lambda$, where $\lambda$ is the density or the PP, is called the bunching function because it describes the bunching effect appearing in the PP obtained in photodetection [4], [8], [9]. It is also sometimes called the intensity function (see [10, p. 69]).

Note that the coincidence function is an even function of $t$ and that it tends, in general, to $\lambda^{2}$ when $t$ tends to infinity. This property comes from the fact that, for large values of $\theta-\theta^{\prime}$, the increments $d N(\theta)$ and $d N\left(\theta^{\prime}\right)$ become, in general, uncorrelated, and their mean value is $\lambda d \theta$. In the case of Poisson processes, these increments are independent, whatever the value of $\theta-\theta^{\prime}$, which implies that the coincidence function is constant and equal to $\lambda^{2}$.

The coincidence function satisfies a relation which will play a fundamental role in what follows. Let $x_{i}^{[n]}$ be the random variable (RV) equal to the distance between a point $t_{i}$ of the PP and the $n$th point posterior to $t_{i}$, or $x_{i}^{[n]}=t_{i+n}-t_{i}$. This positive RV is sometimes called the life time of order $n$. Its distribution function $F_{n}(t)$ is the probability $P\left[x_{i}^{[n]} \leq t\right]$, and the probability density function (pdf) $f_{n}(t)$ of this life time is the derivative of $F_{n}(t)$ with respect to $t$. For simplicity, we assume that $x_{i}^{[n]}$ is a continuous $\mathrm{RV}$ or that $F_{n}(t)$ has a derivative for any $t$. Since $x_{i}^{[n]}>0$, then $f_{n}(t)=0$ when $t<0$. It can be shown (see [10, p. 69]) that the coincidence function satisfies

$$
c(t)=\lambda \sum_{n=1}^{\infty} f_{n}(|t|) .
$$

The principle of the proof is presented in the Appendix. This equation appears in a rather different form in [5] and [6].

This relation is the basis for the estimation of the coincidence function. For this purpose, we use a TAC system which transforms the sequence of random points $t_{i}$ of the PP into a sequence of values $x_{i}=t_{i+1}-t_{i}$. This positive random signal $x_{i}$ completely describes the PP, and any PP generates such a signal $x_{i}$.

Suppose now that we have $M$ observations of $x_{i}, 1 \leq i \leq$ $M$. The problem is to deduce from these observations an estimation of the coincidence function $c(t)$. The measurement of $\lambda$ appearing in (2) is obvious because the density is the inverse of the mean value of the distances $x_{i}$. For the estimation of $c(t)$, the first step is to replace the series (2) by a finite sum of $S$ terms. As a consequence, we arrive at the estimation of the truncated coincidence function $c_{S}(t)=\lambda \sum_{n=1}^{S} f_{n}(|t|)$. The choice of the appropriate value of $S$ will be discussed later.

For a given value of $S$, such that $M / S$ is an integer $N$, and for each $k$ satisfying $1 \leq k \leq S$, we deduce from the observation $x_{i}$ a set of $S$ signals $s_{i}^{[k]}$ defined by

$$
s_{i}^{[k]}=x_{(k-1) N+i}, \quad 1 \leq i \leq N
$$

and zero otherwise. From these signals, we construct a set of $S$ vectors $\mathbf{X}^{[k]}$ with $N$ components defined by

$$
X_{i}^{[k]}=s_{i}^{[k]}+s_{i+1}^{[k]}+\cdots+s_{i+k-1}^{[k]}, \quad 1 \leq i \leq N .
$$

The histogram of these components will clearly yield an estimation of the pdf $f_{k}(t)$. As a consequence, the histogram of the components of the vector $\mathbf{X}=\mathbf{X}^{[1]}+\mathbf{X}^{[2]}+\cdots+\mathbf{X}^{[S]}$ yields an estimation of the truncated bunching function $b_{S}(t)=$ $(1 / \lambda) c_{S}(t)$. More precisely, consider an interval $[t, t+\Delta T]$, and let $n_{k}(t, \Delta T)$ be the number of samples $X_{i}^{[k]}$ recorded in this interval. If $\Delta T$ is sufficiently small, the mean value $\bar{n}_{k}(t, \Delta T)$ of $n_{k}(t, \Delta T)$ is approximately $N f_{k}(t) \Delta T$. Let $n(t, \Delta T)$ be the number of samples of the components of the vector $\mathbf{X}$ recorded in the same interval. From the definition of $\mathbf{X}$ and $c_{S}(t)=\lambda b_{S}(t)$, the mean value $\bar{n}(t, \Delta T)$ of $n(t, \Delta T)$ is

$$
\bar{n}(\Delta T)=b_{S}(t) \Delta T N=b_{S}(t) \frac{M \Delta T}{S} .
$$

The histogram of $\mathbf{X}$ yields for each experiment a value of the recorded number $n(t, \Delta T)$. If $N$ is sufficiently large, this number yields an estimation of $\bar{n}(t, \Delta T)$ and then of $b_{S}(t)$ or $c_{S}(t)$. This procedure necessarily introduces a statistical error analyzed later. Finally, if we are interested only in the shape of the coincidence function, the histogram can be represented in arbitrary units, and the factor $M \Delta T / S$ can be omitted.

Let us now discuss the influence of the various parameters appearing in this measurement. The two most important are the number $N$ of samples recorded and the number $S$ of terms used for the construction of the vector $\mathbf{X}$ or for the approximation of the series (2) by a sum of $S$ terms. On a pure mathematical point of view, the approximation of a function like $c(t)$, which is defined as a series of other functions, requires the discussion of the uniform convergence of the series. There are pdfs for which this uniform convergence is guaranteed, but as the method is used in the case where the pdfs $f_{n}(t)$ of (2) are unknown, it is impossible to introduce in advance more details on this point.

The number $N$ determines the statistical precision of the histogram, and the number $S$ determines the precision of an approximation of a series by a finite sum. These numbers are not completely independent. Indeed, the total number of samples analyzed by the histogram that yields the estimation of $c_{S}(t)$ is $M=S \times N$. It is this number which determines the duration of the measurement or the complexity of the computer analysis. It is often necessary to introduce an upper bound of this number, and in this case, we must choose $N$ and $S$ for a given value of $M$. This introduces a compromise analogous to the one existing between bias and variance in many statistical measurements, for example, spectral analysis. This will be discussed more precisely in the next section.

There are two last parameters appearing in the construction of the histogram yielding the approximation of the coincidence function. The first is the range $\Delta R$ of values of $t$ of the function $c(t)$. This range must be limited for great values of $t$. Indeed, it is clear that $c_{S}(t)$ tends to zero when $t \rightarrow \infty$ because it is a finite sum of pdfs, while, as previously shown, $c(t) \rightarrow \lambda^{2}$. The second is the number of bins of the histograms 
of values belonging to $\Delta R$. Here, there is also a well-known compromise. Too many bins yield a better precision of the approximation of $c_{S}(t)$ but also decrease the number of samples belonging to each bin and, therefore, increase their variance. This compromise between bias and variance in estimation of the pdf of signals has been analyzed in numerous statistical signalprocessing papers.

Finally, it must be noticed that there is a systematic error in the $k-1$ last components of the vector $\mathbf{X}^{[k]}$. In particular, $X_{N}^{[k]}=s_{N}^{[k]}=x_{k N}$, which is not a sum of $k$ terms. This kind of error also appears in all the measurements of correlation functions of random signals. There are ways to correct this error, but this does not play any role in our methods because in our experiments, $N \gg S$.

\section{PERFormances OF THE Method}

There are only a few PPs for which the coincidence function can be explicitly calculated. They will now be used to look at the agreement between theoretical results and those of computer experiments, which yields an indication on the quality of the method.

\section{A. Poisson Processes}

The pdf of the life time of order $n$ of a Poisson process is

$$
f_{n}(t)=\lambda \frac{(\lambda t)^{n-1}}{(n-1) !} \exp (-\lambda t)
$$

Inserting this expression in (2) yields $c(t)=\lambda^{2}$, which illustrates the fact that a Poisson process has no memory. The error when approximating (2) by a finite sum calculated for $\lambda=1$ is then $\epsilon_{S}(t)=1-\exp (-t) \sum_{n=1}^{S} t^{n} / n !$.

By a simple calculation, we see that we can approximate $c(t)$, which is here equal to one, by using ten terms with an error smaller that $10^{-2}$ for $t<4$. With 15 terms this range becomes $t<7$. Similarly by using 20 terms we can approximate $c(t)$ with an error smaller than $10^{-3}$ for $t<9$. This value of $S$ is the greatest used in the experiments described next.

Let us now present experimental results with Poisson processes. A stationary Poisson process is a renewal PP with an exponential pdf. Then, the distances $x_{i}$ between successive points are independent and identically distributed (IID) RVs, and their common pdf is given by (6) with $n=1$. It is easy to generate in a computer experiment such a sequence, which allows us to discuss the performance of the method in terms of the various parameters appearing in its construction. The experiments are realized with a Poisson process of density $\lambda=1$. This implies that $b_{S}(t)=c_{S}(t)$.

In Fig. 1, we present the experimental results obtained when processing $M=2 \cdot 10^{6}$ samples of the life times $x_{i}$. The parameters of these histograms are $S=5, S=10, S=15$, and $S=20$. The widths of the bins of the histograms are $10^{-2}$. This means that there are $2 \cdot 10^{3}$ values recorded in the interval $[0,20]$ of the figure. Three comments must be made.

First, we experimentally verify the fact that the histograms are constant in an interval increasing with $S$. In the last his-

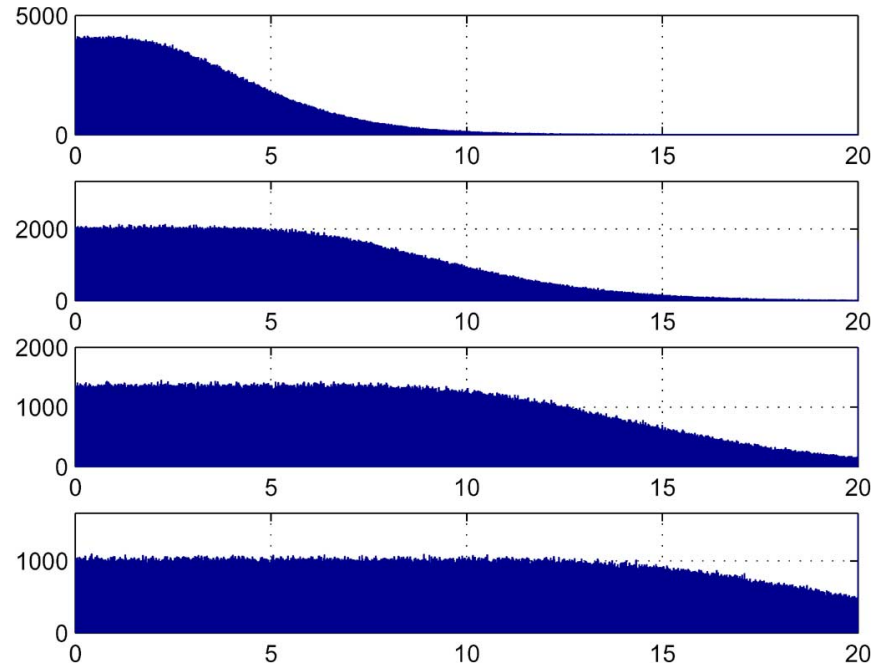

Fig. 1. Coincidence functions of a Poisson process of density 1 measured with $S=5, S=10, S=15$, and $S=20 . M=2 \cdot 10^{6} ; \Delta T=10^{-2}$.

togram obtained for $S=20$, the methods yield excellent results for $t<12$, which means a duration 12 times greater than the mean distance between points of the Poisson process.

Second, as the range of possible values increases with $S$, the statistical precision decreases because the number of samples recorded in each bin decreases. When comparing the first and the last histograms, we observe an increase of the statistical fluctuations. The only way to decrease these fluctuations is to increase the total number $M$ of samples analyzed.

Third, the number of points recorded in each bin corresponds to the values deduced from (5). Applying this expression for $t=0$ and noting that in our experiment $c_{S}(0)=c(0)=1$ because $\lambda=1$, we obtain $\bar{n}(\Delta T)=M \Delta T / S$. In this experiment, $M=2 \cdot 10^{6}$ and $\Delta T=10^{-2}$. The values of $\bar{n}(\Delta T)$ corresponding to the four values of $S$ used are then $4 \cdot 10^{4}$, $2 \cdot 10^{3}, 1.33 \cdot 10^{3}$, and $10^{3}$, which clearly appear on the figure.

\section{B. Erlang Processes}

Erlang processes belong to a class of PPs in which the pdf of the life time of order one is

$$
f_{1}(t)=\mu(\mu t) \exp (-\mu t)
$$

The mean value of the life time is $2 / \mu$, and the density $\lambda$ is then $\mu / 2$. According to (6), this means that $f_{1}(t)$ is the pdf of the life time of order two of a Poisson process of density $\mu$.

Since the pdf (7) does not define a specific PP, there are various different Erlang processes. Their difference comes from the fact that, even if the pdfs of the RVs $x_{i}$ are the same, the other statistical properties are different. There are, of course, a great variety of Erlang processes, depending on the correlation between the $x_{i}$ 's. We shall only consider the two simplest cases.

The first case is the renewal Erlang process. It is the renewal PP defined by the pdf (7). It can be generated by the following procedure. Let $u_{i}$ and $v_{i}$ be the two independent signals, and suppose that they are sequences of IID RVs with the same 
exponential distribution. It is clear that $x_{i}=u_{i}+v_{i}$ is a sequence of IID RVs with the common pdf (7).

In order to calculate the coincidence or the bunching function $b(t)=c(t) / \lambda$, we use the following procedure. The Laplace transform (LT) of (7) is $F(s)=[\mu /(s+\mu)]^{2}$. The assumption that the PP is a renewal process yields that the LT of $f_{n}(t)$ is $F_{n}(s)=[F(s)]^{n}$. Then, the LT of the bunching function is $B(s)=F(s) /[1+F(s)]$ or

$$
B(s)=\frac{\mu^{2}}{(s+\mu)^{2}-\mu^{2}}=\frac{\mu}{2}\left[\frac{1}{s}-\frac{1}{s+2 \mu}\right] .
$$

As a result, the bunching function is

$$
b(t)=\frac{\mu}{2}[1-\exp (-2 \mu t)] .
$$

The second Erlang process is defined as follows. Consider a Poisson process, and let $x_{i}$ be the life time of order one or the distance between successive points. Let $y_{i}=x_{i}+x_{i+1}$. It is clear that $y_{i}$ is the life time of order two of the Poisson process, and its pdf is given by (7). The RVs $y_{i}$, however, are no longer independent because, for example, $x_{i}$ appears in $y_{i}$ and $y_{i-1}$. Consider now the PP defined by the fact that $y_{i}$ is the distance between successive points. It is then an Erlang process but not a renewal process. Changing the origin of time, we can write $y^{[1]}=x_{1}+x_{2}$ and

$$
y^{[n]}=x_{1}+2\left(x_{2}+x_{3}+\cdots+x_{n}\right)+x_{n+1}, \quad n>1
$$

where $x_{i}$ 's are IID RVS with the exponential distribution $\mu \exp (-\mu t)$. We deduce that the LT of the pdf of $y^{[n]}$, which is the life time of order $n$, is

$$
F_{n}(s)=\left(\frac{\mu}{s+\mu}\right)^{2}\left(\frac{\mu}{2 s+\mu}\right)^{n-1}, \quad n \geq 1 .
$$

It is easy to calculate the LT of the bunching function defined by $B(s)=\sum_{n=1}^{\infty} F_{n}(s)$, and its inverse $\mathrm{LT}$ is

$$
b(t)=\frac{\mu}{2}\left[1-\left(1-\frac{t}{\mu}\right) \exp (-\mu t)\right]
$$

which must be compared to (9) obtained previously.

The experimental results for these two Erlang processes appear in Fig. 2. These figures use arbitrary units because we are only interested in the shape of the coincidence function in comparison with its theoretical value. Fig. 2(a) corresponds to the Erlang renewal process. The continuous curves are those deduced from (9) in Fig. 2(a) and (12) in Fig. 2(b), and the experimental points are obtained from an experiment with $10^{6}$ samples and with $\mu=1$ and $S=10$. We see that there is an excellent agreement between theory and experiment. Even if the difference between the two Erlang processes is rather small, it appears clearly.

All the results of this section show the good performance of the method of measurement of the coincidence function introduced in this paper.
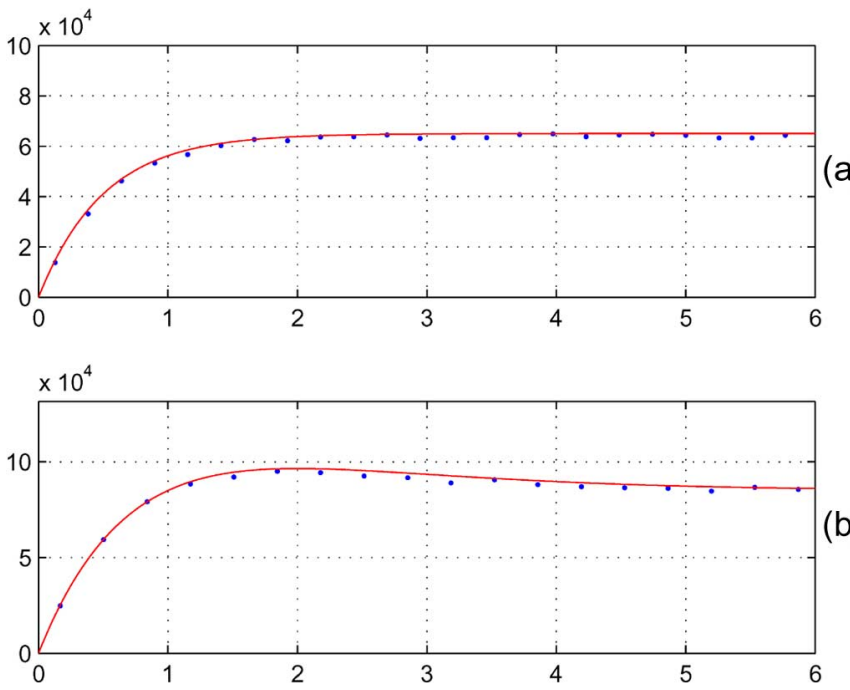

Fig. 2. Coincidence functions of two Erlang processes in arbitrary units. Continuous curves: Theory. Points: Computer experiment.

\section{Poisson Process With Input Dead Time}

Dead-time effects appear in almost all the PPs practically used. Dead time occurs when two points of a PP are so close together that they cannot be registered. This means that some points are erased. If the value $D$ of the dead time is very small compared to the mean distance between points, its effect can be neglected. On the other hand, when the density $\mu$ of the process increases, there is always a value of the density such that dead time must be taken into consideration.

The input dead time is characterized by the fact that each point $t_{i}$ of the PP generates an interval $\left[t_{i}, t_{i}+D[\right.$ such that all the points of the process arriving in this interval are erased. It is clear that when the density $\mu$ increases, the number of points erased increases, and for very large density, almost all the points of the initial PP are erased. This corresponds to the classical congestion phenomenon. Note that the input dead time is sometimes called the type II counter (see [7, p. 101]).

The dead-time effect transforms a given PP into another one. As in our approach, a PP is defined by the life times or by the distance between successive points; the first problem is to calculate the life time after dead time in terms of the life time of the initial PP. The explicit expression of the result is almost impossible to obtain in closed form. A recursive algorithm, however, has been introduced and analyzed in [11]. This algorithm is used for the estimation of the coincidence function when the initial PP is a Poisson process. In this case, the theoretical calculation of the coincidence function with input dead time is particularly simple. For this, it suffices to start from (1). If $\theta-\theta^{\prime}<D$, the expectation is zero because one of the two points is necessarily erased. Otherwise, $c(t)$ is constant, and its value is $\lambda^{2}$. Note that $\lambda$ is the density of the PP with dead time. If the density of the initial Poisson process is $\mu$, a simple calculation yields that $\lambda=\mu \exp (-\mu D)$. This expression explains the congestion phenomenon indicated previously because $\lambda$ tends to zero when $D$ tends to infinity. In the opposite case, $\lambda=\mu$ if $D=0$. 

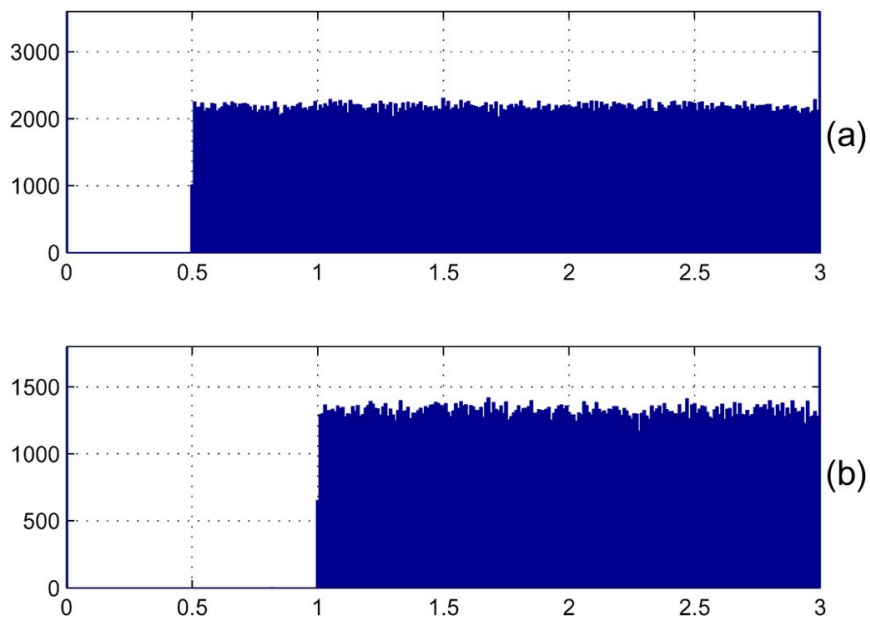

Fig. 3. Coincidence functions of a Poisson process with unit density with input dead time. (a) $D=0.5$. (b) $D=1$.

Experimental results are shown in Fig. 3. The density of the Poisson process is $\mu=1$, and two values of the dead time are used: 0.5 and 1 . The number $S$ of terms in the sum defining $c(t)$ is ten, which ensures good precision of the results for $t<3$. The fact that $c(t)=0$ for $t<D$ is well verified. On the other hand, the fact that $c(t)$ is constant for $t>D$ is also verified, although statistical fluctuations of the estimation remain. These fluctuations are more important for $D=1$ than for $D=0.5$ because more points are erased in the second case than in the first. Finally, as in these experiments, $M=3.6 \cdot 10^{6}, \Delta T=$ $10^{-2}$, and $S=10$, and the application of (5) with $b_{S}(t)=$ $\mu \exp (-\mu D)$ yields that the mean number of points $\bar{n}(\Delta T)$ in each bin is for $t>D$ equal to 2183 or 1324 for the cases 1 or 2 , respectively. This is very well verified in the two histograms in Fig. 3.

\section{AnAlysis of Some Particular PPS}

In this section, we shall analyze the second-order properties of some PPs interesting in various applications but for which theoretical analysis is difficult, which justifies the experimental approach.

\section{A. Poisson Process With Output Dead Time}

This dead time, which is also called the type I counter (see [7, p. 101]), is characterized by the fact that each point $\theta_{i}$ of the Poisson process, which is not erased, generates an interval $\left[\theta_{i}, \theta_{i}+D\left[\right.\right.$ such that all the points $t_{i}$ of the PP in this interval are erased. This means that only the points not erased contribute to the dead time. In this case, when $\mu \rightarrow \infty$, the PP after dead time becomes a periodic process or a sequence of points with equal distances $D$.

The calculation of the life time after output dead time in terms of the life time of the initial PP is very complicated. As previously stated, however, a recursive algorithm for this purpose has been introduced in [11]. We shall use this algorithm for the estimation of the coincidence function after an output dead-time effect when the input is a Poisson process.

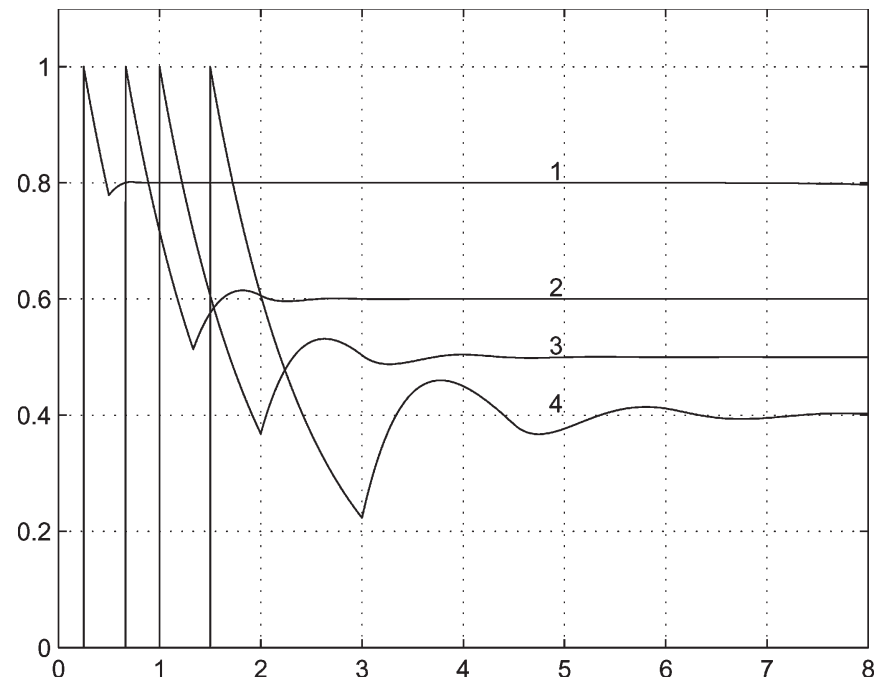

Fig. 4. Bunching function of a Poisson process with unit density and output dead time. (1) $D=1 / 4$, (2) $D=2 / 3$, (3) $D=1$, and (4) $D=3 / 2$.

Let us first present how this coincidence function can be calculated in this case. It is easy to see that the PP after dead time is a renewal process entirely defined by the pdf of its life time. This pdf is

$$
f(t)=u(t-D) \mu \exp [-\mu(t-D)]
$$

where $u($.$) is the unit step function, D$ is the value of the dead time, and $\mu$ is the density of the Poisson process. It results from this expression that the mean value of the life time after dead time is $D+1 / \mu$. This quantity is the inverse of the density which is then $\lambda=\mu /[1+\mu D]$. We observe that when $\mu \rightarrow \infty, \lambda \rightarrow D$, which was indicated previously. Furthermore, when $D \rightarrow \infty, \lambda \rightarrow 0$, which appears with any dead-time effect.

As the PP analyzed is a renewal process, its pdf $f_{n}(t)$ is deduced from (13) by $n$ convolutions, and the result is

$$
f_{n}(t)=u(t-n D) \mu \frac{[\mu(t-n D)]^{n-1}}{(n-1) !} \exp [-\mu(t-n D)] \text {. }
$$

In order to calculate the coincidence function, we can use (2). There is no explicit analytic expression for $c(t)$, but its numerical calculation is possible. Indeed, because of the term $u(t-n D)$ in (14), the series is always limited to a finite sum. As we must have $t-n D>0$, the number of terms in this sum is the greatest integer smaller than $t / D$.

The numerical results of calculations are shown in Fig. 4 where some examples of the bunching function $b(t)=$ $(1 / \lambda) c(t)$ are presented. The value of $\mu$ is one, and the values of $D$ are $1 / 4,2 / 3,1$, and $3 / 2$ in such a way that the corresponding values of the density $\lambda$ are $0.8,0.6,0.5$, and 0.4 . We verify that $b(t) \rightarrow \lambda$ when $t \rightarrow \infty$. In reality, the values of $t$ are limited to the interval $0<t<8$, and for this interval, the sum (2) is truncated to 12 terms.

We see on this figure that when $D=1 / 4$, the effect of the dead time on the coincidence function disappears when $t>$ $2 / 3$. For $D=1$, this inequality becomes $t>4$. Furthermore, 

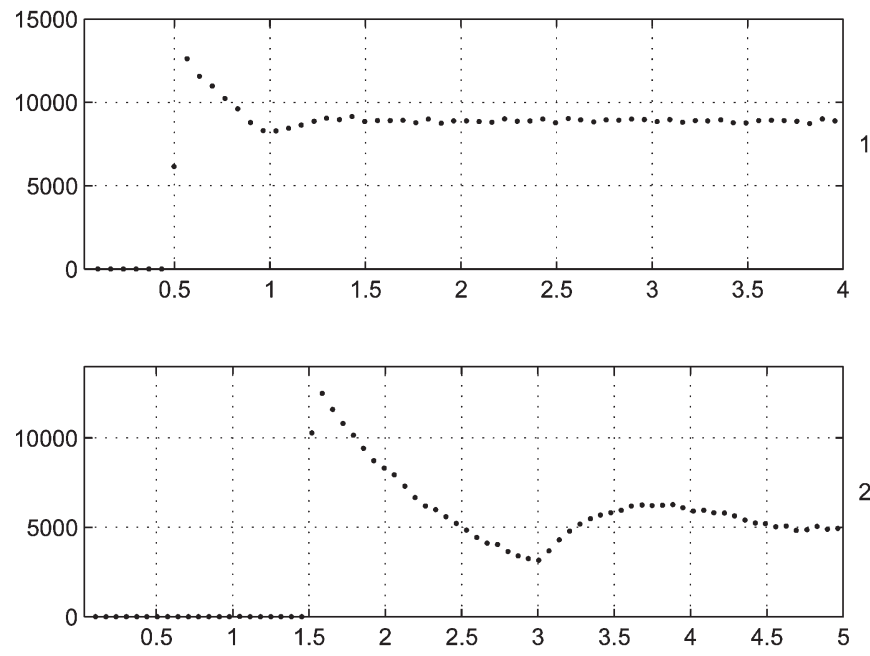

Fig. 5. Coincidence functions of a Poisson process with unit density and output dead time. (1) $D=0.5$. (2) $D=1.5$.

all these curves show a discontinuity of the derivative for the value $t=2 D$, which is the time at which the second term $f_{2}(t)$ appears in the sum (2).

The experiments with the output dead time are much more difficult. Indeed, the algorithm yielding the life time after dead time presented in [11] introduces much more complexity and calculation time. This limits the possible values of $M$ and, thus, the statistical precision of the procedure. This effect is increased by the fact that, as in any experiment with dead time, many points are erased, and the number of those playing a role in the estimation tends to zero when $D$ increases.

The experimental results appear in Fig. 5. Estimations of the coincidence function are presented for $D=0.5$ and $D=1.5$. The results are in a rather good agreement with those in Fig. 4, but some statistical fluctuations remain. Their suppression would require more memory and computer time.

\section{B. Renewal Process With Uniform Distribution}

Consider a renewal PP in which the pdf $f(t)$ of the distance between successive points is a rectangular function equal to $1 / 2 b$ when $t$ is in the interval $[m-b, m+b]$ and zero otherwise. This kind of pdf appears in the description of jitter phenomena in which the distance between successive points is not strictly constant, which appears if $b \rightarrow 0$. The mean value of the distance is clearly $m$, and as a result, the density $\lambda$ of the corresponding $\mathrm{PP}$ is $1 / \mathrm{m}$.

The pdfs $f_{n}(t)$ can be calculated by successive convolutions. The calculation is possible but rapidly becomes very complicated, and no explicit expression of the convolution is available. Two points, however, can be noted. First, the pdf $f_{2}(t)$ of the second-order life time is a triangular function equal to $\left(1 / 4 b^{2}\right)[-|t-2 m|+2 b]$ for $|t-2 m|<2$, and zero otherwise. In particular, $f_{2}(2 m)=1 / 2 b$. Second, when $n \gg 1$, $f_{n}(t)$ tends to have a Gaussian shape of the form $N\left(n m, n \sigma^{2}\right)$, where $\sigma^{2}$ is the variance associated with the pdf $f(t)$ and equal to $b^{2} / 3$.

Experimental results are shown in Fig. 6. The bunching function of the PP is estimated for the values $m=1$. The values

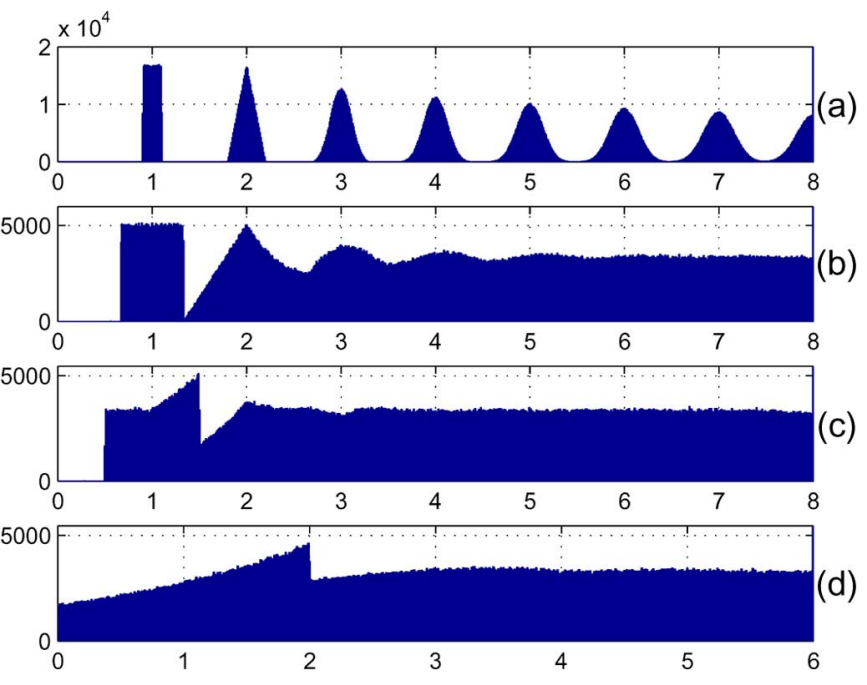

Fig. 6. Coincidence functions of a renewal process with uniform distribution. Values of $b: 0.1,1 / 3,1 / 2$, and 1 .

chosen for $b$ are $1 / 10,1 / 3,1 / 2$, and 1 . The number of samples used is $M=3 \cdot 10^{6}$, the number of terms in (2) is $S=9$, and the width of the bins is $\Delta T=10^{-2}$. As a consequence, the coefficient $M \Delta T / S$ in (5) is $(1 / 3) 10^{4}$.

These functions suggest the following comments. We note first that if $b \rightarrow 0$, the PP tends toward a periodic PP, which means a periodic distribution of points with a period equal to one. This is easily verified experimentally but has no practical interest. The value $b=0.1$ is still rather small, and a memory of the periodic structure remains. The different pdfs $f_{n}(t)$ appearing in the figure do not overlap, at least, for $t<8$ used in Fig. 6(a). We clearly observe the rectangular and triangular functions $f_{1}(t)$ and $f_{2}(t)$. For large values of $t$ not represented in the figure, the Gaussian shapes overlap in such a way that their sum becomes constant and equal to the asymptotic value one of $b(t)$. This, however, requires very great values of $S$ and $M$, which cannot be obtained in our experiments.

Fig. 6(b) corresponds to $b=1 / 3$. This value has been chosen because it is the greatest value of $b$ for which $f_{1}(t)$ and $f_{2}(t)$ do not overlap. The corresponding limit point is $t=1+1 / 3$, which clearly appears on the figure. For this value of $b$, the rectangular function $f_{1}(t)$ is equal to $3 / 2$. Applying (5) with the value calculated previously yields $(1 / 2) 10^{4}=5000$, which clearly appears in Fig. 6(b). The same can be said for $f_{2}(2)$ which has the same value. Finally, for $t \rightarrow \infty$, the bunching function tends to one, which yields a number of samples in each bin of $(1 / 3) 10^{4}$, which appears in Fig. 7(b)-(d).

The theoretical calculation of the bunching function corresponding to the values used in Fig. 6(c) and (d) is almost impossible. This justifies the interest of the experimental results displayed on these figures.

\section{PP With Uniform Distribution and Correlated Life Times}

In this section, we assume that the pdf of the life time is still rectangular, as in the previous one, but the PP is no longer a renewal PP. This means that the distances between successive 

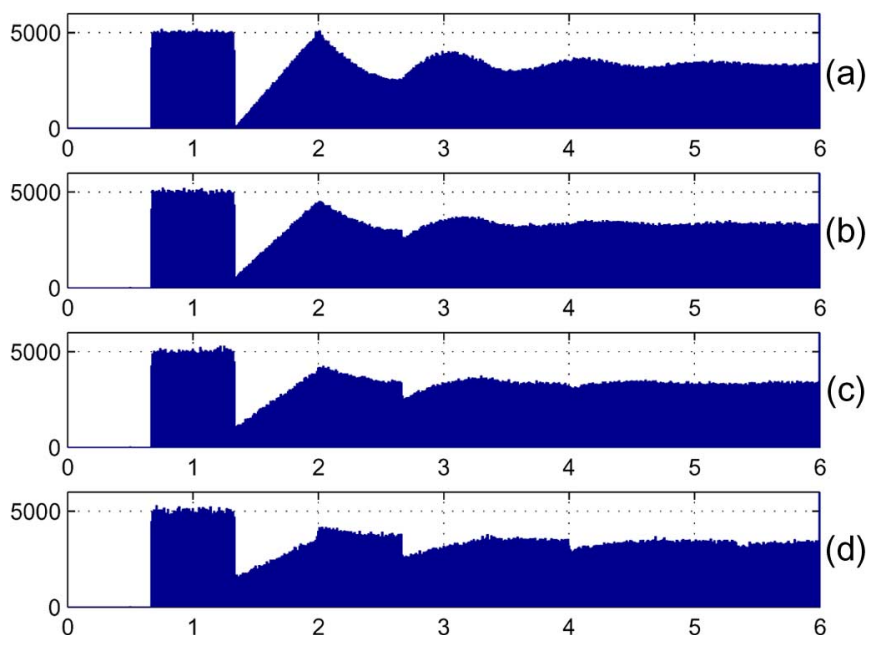

Fig. 7. Coincidence functions of $\mathrm{PP}$ with uniform distribution and correlation. $m=1 ; b=1 / 3$. Values of $p: 0,0.2,0.4$, and 0.6 .

points are identically distributed (rectangular distribution) but no longer independent.

An algorithm for generating this kind of life time has been presented in [11], and its principle is outlined in the Appendix. The normalized correlation function of the first-order life time is exponential or $p^{|n|}$. If $p=0$, we find again the case of the previous section.

The experimental results are shown in Fig. 7. The parameters of this figure are the same as in Fig. 6(b), and our objective is only to evaluate the effect of the correlation characterized by the parameter $p$. Fig. 7(a) corresponds to $p=0$, the same situation as in Fig. 6(b), and we find again the same form of coincidence function. When $p$ increases, this coincidence function is strongly modified, and the theoretical calculations are impossible because of the complexity of the model. However, the effect of a correlation is perfectly clear in Fig. 7(b)-(d). In all these figures, $f_{1}(t)$ is the same, and this appears for values of $t$ smaller than 1.333. We have then a good example of PPs with the same distribution of life time and entirely different secondorder properties characterized by the coincidence functions shown in Fig. 7.

\section{CONCLUSION}

The second-order properties of a PP are entirely contained in its coincidence function. In this paper, we have shown that this function can be measured by an appropriate processing of the distance between successive points obtained, for example, from a TAC. The theoretical foundation of this processing is an expression that yields a relation between the coincidence function and the pdf of the life time of the PP. For its practical realization, we replace a series by a sum of a finite number of terms constructed from appropriate histograms of samples of the signal. The method has been tested in the case of PPs where the coincidence function has an explicit theoretical expression, as in the case of Poisson processes with or without dead time, as well as in the case of some Erlang PPs. The experimental results are in excellent agreement with the theoretical calculations. Finally, when this method is used for the measurement of coincidence functions that cannot be obtained theoretically, the experimental results exhibit various interesting features.

\section{APPENDIX}

The expectation (1) defining the coincidence function is equal to the probability $P\left\{[d N(\theta)=1] \cap\left[d N\left(\theta^{\prime}\right)=1\right]\right\}$. This probability can be written as

$$
\begin{aligned}
& P\left\{[d N(\theta)=1] \cap\left[d N\left(\theta^{\prime}\right)=1\right]\right\} \\
& \quad=P[d N(\theta)=1] \cdot P\left\{\left[d N\left(\theta^{\prime}\right)=1\right] \mid[d N(\theta)=1]\right\} .
\end{aligned}
$$

We deduce from the definition of the density $\lambda$ that $P[d N(\theta)=$ $1]=\lambda d \theta$. The pdf $f_{n}\left(\theta-\theta^{\prime}\right)$ is defined by

$f_{n}\left(\theta-\theta^{\prime}\right) d \theta^{\prime}=P\left\{\left[d N\left(\theta^{\prime}\right)=1\right] \mid A_{n}\left(\theta^{\prime}-\theta\right) \cap[d N(\theta)=1]\right\}$

where $A_{n}\left(\theta^{\prime}-\theta\right)$ is the event that there are $n-1$ points of the $\mathrm{PP}$ in $\left[\theta+d \theta, \theta^{\prime}[\right.$. It is clear that

$$
\begin{aligned}
& P\left\{\left[d N\left(\theta^{\prime}\right)=1\right] \mid[d N(\theta)=1]\right\} \\
& \quad=\sum_{n=1}^{\infty} P\left\{\left[d N\left(\theta^{\prime}\right)=1\right] \mid A_{n}\left(\theta^{\prime}-\theta\right) \cap[d N(\theta)=1]\right\} .
\end{aligned}
$$

This yields (2).

The statistical signal used in Section IV-C is constructed as follows. Let $u_{k}$ and $v_{k}$ be the two independent IID RVs. Suppose furthermore that $u_{k}$ takes only the value zero or one, and let $p$ be the probability that $u_{k}=1$. Let $f($.$) be the pdf of$ the $v_{k}$ 's. Consider now the signal $x_{k}=u_{k} x_{k-1}+\left(1-u_{k}\right) v_{k}$. It is clear that if the pdf of $x_{1}$ is $f($.$) , all the other x_{k}$ 's have the same pdf. It can be shown that this is asymptotically verified, whatever the pdf of $x_{1}$. The $x_{k}$ 's have then the same pdf but are correlated. Their correlation function $\gamma_{k}$ satisfies the recursion $\gamma_{k}=p \gamma_{k-1}$. This yields $\gamma_{k}=\sigma^{2} p^{k}$, where $\sigma^{2}$ is the variance of the RVs $v_{k}$. For $p=0, x_{k}=v_{k}$, whereas for $p=1$, all the RVs $x_{i}$ 's are equal.

\section{REFERENCES}

[1] M. Bartlett, "The spectral analysis of point processes," J. R. Stat. Soc., B, vol. 25, no. 2, pp. 264-296, 1963.

[2] M. Bartlett, An Introduction to Stochastic Processes. Cambridge, U.K.: Cambridge Univ. Press, 1966.

[3] O. Macchi, "The coincidence approach to stochastic point processes," Adv. Appl. Probab., vol. 7, no. 1, pp. 83-122, Mar. 1975.

[4] B. Picinbono and C. Bendjaballah, "Coincidence analysis of point processes," in Proc. EUSIPCO, 2004, pp. 1063-1066.

[5] V. Kryukov, "Calculation of the correlation function and the spectral power density of random sampling," Radio Eng. Electron. Phys., vol. 12, pp. 169-176, 1967.

[6] J. Beutler and O. Leneman, "The spectral analysis of impulse processes," Inf. Control, vol. 12, no. 3, pp. 236-258, 1968.

[7] D. R. Cox and V. Isham, Point Processes. London, U.K.: Chapman \& Hall, 1980.

[8] B. Picinbono and C. Bendjaballah, "Characterization of nonclassical optical fields by photodetection statistics," Phys. Rev. A, Gen. Phys., vol. 71, no. 1, pp. 013 812-1-013 812-12, 2005.

[9] B. Saleh, Photoelectron Statistics. Berlin, Germany: Springer-Verlag, 1977.

[10] D. R. Cox and P. A. W. Lewis, The Statistical Analysis of Series of Events. London, U.K.: Methuen, 1966.

[11] B. Picinbono, Dead Time Effects in Point Processes, 2006. L2S internal report. 


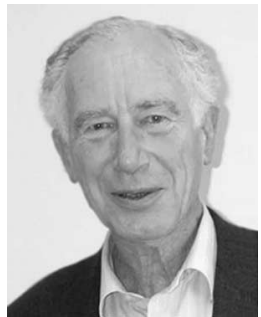

Bernard Picinbono (M'63-SM'79-F'85-LF'00) was born in Algiers, Algeria, on March 21, 1933. He received the Agrégation des Sciences Physiques degree in 1956 and the Doctorat d'État degree in 1960.

From 1960 to 1965 , he was a Professor with the Department of Physics, University of Algiers. In 1965, he joined the University of Paris-Sud (UPS), Orsay, Paris, France, to which he is still attached (Emeritus). He was the President of this university between 1971 and 1975 and the Director of the Laboratoire des Signaux et Systèmes (L25), which is a joint research laboratory of the Centre National de Ia Recherche Scientifique and the École Supérieure d'Électricité (ESE) and is associated with the UPS, between 1975 and 1987. Between 1990 and 1995, he was the General Director of the ESE. His research interests include statistical signal processing, detection and estimation, and stochastic processes. He is the author of numerous papers and books on these subjects.

Dr. Picinbono is a member of the French Academy of Sciences and of the Académie des Technologies. He was the President of the GRETSI Conference organized every two years in France and devoted to signal and image processing problems. He was the cochairman of the 1982 IEEE International Symposium of Information Theory, Les Arcs, France. 